EDITORIAL

\title{
La soja como tratamiento complementario de los síntomas de la menopausia
}

\section{Soy As a Complementary Treatment for Menopause Symptoms}

\author{
Nahyr Schinca ${ }^{a}$ e Iva Marques-Lopes ${ }^{\mathrm{b}, *}$ \\ aAsociación Española de Dietistas-Nutricionistas \\ 'Universidad de Zaragoza
}

Recibido el 10 de abril de 2012; aceptado el 16 de abril de 2012

El aumento de la esperanza de vida en los últimos años ha conllevado que un mayor número de mujeres alcance los 50 años en condiciones que les permitan disfrutar de la etapa de la menopausia con mejor salud física y mental.

Entre los factores que afectan a la calidad de vida de la mujer en esta etapa fisiológica, se encuentran los relacionados con los efectos secundarios del cambio hormonal que se produce durante la menopausia, lo cual se puede controlar mediante diferentes aproximaciones terapéuticas, incluidas la alimentación y el estilo de vida.

En este contexto, la utilización de las isoflavonas de la soja se considera entre los tratamientos complementarios que podrían ayudar a disminuir algunos de sus síntomas. Considerando la complejidad de los síntomas en la menopausia, y dejando de lado los sofocos, la relación entre la utilización de las isoflavonas de la soja y la mayoría de los síntomas de la menopausia se ha estudiado poco o nada. Cabe destacar, sin embargo, los sofocos, que afectan a la gran mayoría de las mujeres menopáusicas, y de los que se han realizado más estudios científicos al respecto. Un reciente metaanálisis - publicado en la revista Menopause ${ }^{1}$ después de que la revisión que aparece en el presente número de Revista Española de Nutrición Humana y Dietética (RENHDIET) se aceptara para publicación- concluye que los suplementos de isoflavonas disminuyen significativamente la

*Autor para correspondencia.

Correo electrónico: imarques@unizar.es (I. Marques-Lopes). frecuencia y la intensidad de los sofocos. Asimismo, el consumo de isoflavonas de la soja parece mejorar la calidad de vida de la mujer menopáusica y posmenopáusica más allá de su efecto en los sofocos, ya que se encuentra asociada a una disminución del riesgo de padecer afecciones relacionadas con la densidad mineral ósea ${ }^{2}$ y con la salud cardiovascular ${ }^{3}$. En este sentido, y pese a que no hay suficiente evidencia científica para establecer una recomendación de nivel A para la ingesta de las isoflavonas de la soja para todos los síntomas y/o las enfermedades asociadas a esta etapa del ciclo vital, tampoco se han detectado posibles efectos adversos de su utilización, por lo que su recomendación como terapia complementaria podría coadyuvar a la terapia médica convencional.

Si bien los efectos de los extractos de isoflavonas de la soja han sido más estudiados, son muchos los estudios que tratan de averiguar hasta qué punto el consumo de soja a través de la dieta puede ser eficaz en la disminución efectiva de los síntomas de la menopausia, así como de algunas enfermedades asociadas, como por ejemplo la osteoporosis, la enfermedad cardiovascular o el cáncer dependiente de hormonas. Pese a que hay varios artículos que parecen poner de manifiesto posibles beneficios de la utilización de la soja para algunos síntomas menopáusicos ${ }^{4}$, los estudios de intervención realizados indican que es necesario ingerir grandes cantidades de soja para garantizar una concentración adecuada de isoflavonas, y los datos de estudios observacionales indican que la cantidad de soja que se puede consumir a través de la dieta, incluyendo varios productos 
derivados o elaborados a base de soja, podría no garantizar la suficiente concentración de isoflavonas que permita aliviar de forma significativa los trastornos asociados a la menopausia ${ }^{5}$.

Actualmente diversas sociedades científicas, entre ellas la Sociedad Española de Ginecología y Obstetricia (SEGO) y la Asociación Española para el Estudio de la Menopausia (AEEM), incluyen la ingesta de los derivados de la soja como tratamiento complementario que podría disminuir algunos síntomas menopáusicos.

En este número de RENHDIET, se presenta un interesante artículo de revisión sobre el estado de la evidencia científica de la posible acción terapéutica y beneficiosa de las isoflavonas de soja y su potencial estrogénico, en el que sus autoras nos detallan las conclusiones que pueden extraerse actualmente. Entre las conclusiones de las autoras, cabe destacar:

- Por un lado, si bien actualmente hay una mayor aceptación e inclusión de la soja en los hábitos alimentarios de nuestra población, la ingesta diaria de isoflavonas de las mujeres españolas es escasa y sigue siendo mucho más baja (< $1 \mathrm{mg} /$ día $)^{4}$ si se la compara con la ingesta diaria de las mujeres orientales ( $60 \mathrm{mg} /$ día), en las que se han realizado más estudios y se han observado efectos más significativos ${ }^{6}$. Este hecho impide trasladar los hallazgos de estudios realizados en población oriental, lo que es un problema de difícil solución, porque implica un importante cambio de hábitos alimentarios de un subgrupo de población.

- Los efectos fisiológicos de las isoflavonas de la soja podrían trasladarse a los efectos de la ingesta de la soja y sus derivados, siempre y cuando las cantidades presentes en los alimentos fueran similares a las utilizadas en dichos estudios7. Sin lugar a dudas, un aspecto en el que la industria alimentaria puede tener un importante papel.

Se trata, por lo tanto, de un tema de rabiante actualidad, de candente discusión científica y, evidentemente, de gran trascendencia para la calidad de vida de las mujeres que sigue pendiente de nuevas proyecciones sanitarias.

Aprovechando que en marzo, momento en el que se escribe el presente editorial, fue el Día Internacional de la Mujer, y que este editorial está dedicado a un tema importante de la salud y la calidad de vida de la mujer, creemos que es el momento ideal para anunciar los cambios que se están realizando en el Comité Editorial de RENHDIET, más aún cuando dichos cambios implican a las dos mujeres firmantes.

RENHDIET, llamada en sus inicios Actividad Dietética, se concibió en 1997, cuando el laboratorio de Nutrición Wander, hoy Nestlé, propuso y potenció esta idea, hecho que se ha agradecido desde el principio. La remodelación de la Revista, iniciada en 2008, con el nuevo Comité y Consejo
Editorial, tuvo y tiene como objetivo principal la adaptación a las normas del Science Citation Index de Thomson Reuters, que permitirá la valoración del Impact Factor y dará lugar a su indexación en bases de datos electrónicas especializadas, lo que le dará el reconocimiento científico que anhelamos y se merece.

Esta es una nueva etapa que se cursará con orgullo para la Asociación Española de Dietistas-Nutricionistas (AEDN), porque la Revista sigue y seguirá adelante con paso firme.

Es un enorme placer presentar a la nueva Editora Jefe de RENHDIET, la Dra. Iva Marques-Lopes, dietista-nutricionista, doctora en Fisiología y Alimentación, profesora titular de la Universidad de Zaragoza, Presidenta de la Conferencia Española de Decanos y Directores que imparten la titulación de Nutrición Humana y Dietética, y miembro del grupo de investigación de excelencia en Dislipemias del Gobierno de Aragón. Estamos, sin lugar a dudas, ante una persona cuyas situación personal, trayectoria científica y calidad humana merecen el máximo respeto, y así le damos una cálida bienvenida agradeciéndole el relevo.

También es de agradecer a todos los/las Dietistas-Nutricionistas, a todos los profesionales sanitarios que se dedican, como docentes e investigadores, a la Nutrición Humana y Dietética, y a los lectores/as y demás profesionales que a lo largo de los 15 años compartieron con nosotros uno u otro aspecto de esta revista.

\section{Bibliografía}

1. Taku K, Melby MK, Kronenberg F, Kurzer MS, Messina M. Extracted or synthesized soybean isoflavones reduce menopausal hot flash frequency and severity: systematic review and metaanalysis of randomized controlled trials. Menopause. $2012 \mathrm{Mar}$ 19 [Epub ahead of print].

2. Taku K, Melby MK, Kurzer MS, et al. Effects of soy isoflavone supplements on bone turnover markers in menopausal women: systematic review and meta-analysis of randomized controlled trials. Bone. 2010;47:413-23.

3. Li SH, Liu XX, Bai YY, et al. Effect of oral isoflavone supplementation on vascular endothelial function in postmenopausal women: a meta-analysis of randomized placebo-controlled trials. Am J Clin Nutr. 2010;91:480-6.

4. Cassidy A, Albertazzi P, Lise Nielsen I, et al. Critical review of health effects of soyabean phyto-oestrogens in post-menopausal women. Proc Nutr Soc. 2006;65:76-92.

5. González CA. Estimation of dietary sources and flavonoid intake in a Spanish adult population (EPIC-Spain). J Am Diet Assoc. 2010;110:390-8.

6. Cornwell T, Cohick W, Raskin I. Dietary phytoestrogens and health. Phytochemistry. 2004;656:995-1016.

7. Vergne S, Titier K, Bernard V, et al. Bioavailability and urinary excretion of isoflavones in humans: effects of soy-based supplements formulation and equol production. J Pharm Biomed Anal. 2007;43:1488-94. 